\title{
A round-up of recent papers in the field of photonics published by the physical sciences division of the Nature Publishing Group.
}

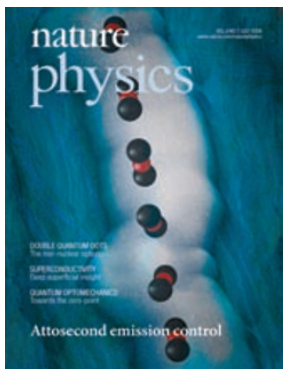

Universal emission intermittency

in quantum dots, nanorods and nanowires

\section{Nature Phys. 4, 519-522 (2008)}

Virtually all known fluorophores exhibit mysterious episodes of emission intermittency. A remarkable feature of the phenomenon is a power-law distribution of on- and off-times observed in colloidal semiconductor quantum dots, nanorods, nanowires and some organic dyes. For nanoparticles, the resulting power law extends over an extraordinarily wide dynamic range: nine orders of magnitude in probability density and five to six orders of magnitude in time. Exponents hover about the ubiquitous value of $-3 / 2$. Dark states routinely last for tens of secondspractically forever on quantum mechanical timescales. Despite such infinite states of darkness, the dots miraculously recover and start emitting again. Although the underlying mechanism responsible for this phenomenon remains a mystery and many questions persist, we argue that substantial theoretical progress has been made.

\section{Coherent control of attosecond emission from aligned molecules}

\section{Nature Phys. 4, 545-549 (2008)}

Controlling attosecond electron wave packets and soft X-ray pulses represents a formidable challenge of general implication to many areas of science. A strong laser field interacting with atoms or molecules drives ultrafast intra-atomic/molecular electron wave packets on a subfemtosecond timescale, resulting in the emission of attosecond bursts of extreme-ultraviolet light. Controlling the intra-atomic/molecular electron dynamics enables steering of the attosecond emission. Here, we carry out a coherent control in linear molecules, where the interaction of the laser-driven electron wave packet with the core leads to quantum interferences. We demonstrate that these interferences can be finely controlled by turning the molecular axis relative to the laser polarization, that is, changing the electron recollision angle. The wave-packet coulombic distortion modifies the spectral phase jump measured in the extreme-ultraviolet emission. Our attosecond control of the interference results in attosecond pulse shaping, useful for future applications in ultrafast coherent control of atomic and molecular processes.

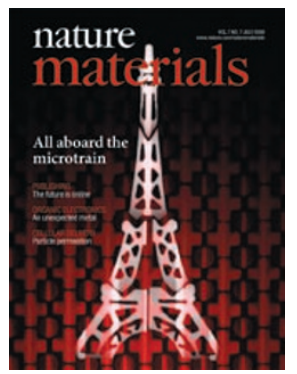

resonators is in good agreement with theory. Retrieval of the effective optical parameters reveals the importance of bi-anisotropy. Once suitable theoretical blueprints are available, our fabrication approach will enable rapid prototyping of truly $3 \mathrm{D}$ photonic metamaterials.

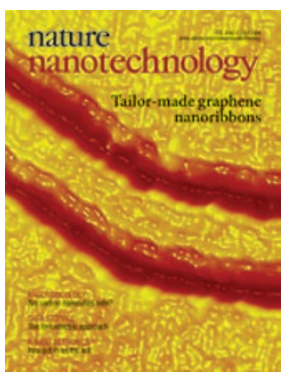

Subwavelength direct-write nanopatterning using optically trapped microspheres

Nature Nano. 3, 413-417 (2008)

A number of non-lithographic techniques are now available for processing materials on the nanoscale, including optical techniques capable of producing features that are much smaller than the wavelength of light used. However, these techniques can be limited in speed, ease of use, cost of implementation, or the range of patterns they can write. Here we report how Bessel beam laser trapping of microspheres near surfaces can be used to enable near-field direct-write subwavelength nanopatterning. Using the microsphere as an objective lens to focus the processing laser, we demonstrate arbitrary patterns and individual features with minimum sizes of $\sim 100 \mathrm{~nm}$ (which is less than one-third the processing wavelength) and a positioning accuracy better than $40 \mathrm{~nm}$ in aqueous and chemical environments. Submicron spacing is maintained between the nearfield objective and the substrate without active feedback control. If implemented with an array of optical traps, this approach could lead to a high-throughput probebased method for patterning surfaces with subwavelength features.

If citing these articles, please reference the complete version in the appropriate journal rather than Nature Photonics. 